\title{
Transformation of polyethylene into a vitrimer by nitroxide radical coupling of a bis-dioxaborolane
}

Received 00th January 20xx, Accepted 00th January 20xx DOI: $10.1039 / \times 0 \times x 00000 x$

\begin{abstract}
Florent Caffy and Renaud Nicolaÿ*
Vitrimers are a new class of polymeric materials that combine the re-processability of thermoplastics with the superior chemical and thermo-mechanical properties of thermosets. Transforming existing thermoplastics, and especially commodity plastics, into vitrimers during their processing is an exciting challenge that could greatly facilitate the industrial development of these materials. In this study, we combined nitroxide chemistry, for radical grafting, and boronic ester metathesis, as an associative exchange reaction, to prepare PE vitrimers via a single-step reactive extrusion of commercial HDPE. After studying the grafting efficiency of a model nitroxide carrying a dioxaborolane moiety, a bis-dioxaborolane bearing two nitroxide functions was used to synthesize vitrimers. The functionalization degree and its impact on the degree of crystallinity of vitrimers were quantified. The flow properties, thermal stability and thermo-mechanical properties of the resulting vitrimers were also studied. We found that associative exchange reactions allow full stress relaxation and provide processability to the vitrimers up to approximately $200^{\circ} \mathrm{C}$. Above this temperature, the thermolabile alkoxyamine bonds within the cross-links irreversibly dissociate, resulting in a material with a zero-shear viscosity comparable to that of the starting HDPE. The recyclability of these vitrimers was successfully exemplified with successive cycles of processing and mechanical testing, while their creep resistance at $80^{\circ} \mathrm{C}$ was compared to that of their HDPE precursor.
\end{abstract}

\section{Introduction}

Thermosets present superior thermo-mechanical properties and chemical resistance as compared to thermoplastics. However, chemically cross-linked polymers cannot be processed multiple times and are therefore not recyclable, unlike thermoplastics. ${ }^{1,2}$ When comparing thermoplastics and thermosets of identical chemical nature, their differences in properties result solely from the modification of their topology. Thermoplastics are constituted of polymer chains of finite sizes, while thermosets are made of a permanent polymer network of infinite dimension. In order to combine the processability of thermoplastics with the superior properties of thermosets, significant efforts have been made in recent years to design chemical networks incorporating reversible and/or dynamic cross-links. ${ }^{2-6}$ While polymer networks based on reversible cross-links undergo partial dissociation at high temperature (or under light irradiation), thereby passing below the gel point to yield polymer chains of finite sizes, networks relying on associative exchange reactions maintain a constant connectivity regardless of the temperature. The ability of the latter to be processed multiple times is made possible by the reshuffling of dynamic bonds within the network, which exchange the fragments they are connecting while maintaining constant the number of chemical bonds in the system. As a result, these

Chimie Moléculaire, Macromoléculaire, Matériaux. ESPCI Paris, CNRS, PSL University, 75005 Paris, France

Electronic Supplementary Information (ESI) available: [details of any supplementary information available should be included here]. See DOI: 10.1039/x0xx00000x materials, coined Vitrimers by Leibler et col.,7,8 present a unique set of properties that make them a class of materials on their own.

The pivotal element of vitrimers is their dynamic crosslinks. Today, a large number of associative exchange reactions have been successfully implemented to design vitrimers and vitrimer-like materials, including transesterification of esters and boronic esters, 7,9 addition-fragmentation chain transfer, ${ }^{10,11}$ transamination of imines and vinylogous urethanes, 12,13 olefin and dioxaborolane metathesis, ${ }^{14,15}$ transcarbonation, ${ }^{16}$ transalkylation and transalkoxylation, ${ }^{17-19}$ addition- elimination of thiols with Meldrum's acid. ${ }^{20}$ However, while the vast majority of the 335 million tons of plastics produced in the world in 2016 were made from olefins or vinyl monomers polymerized via a chain growth process, vitrimers have been so far mostly prepared by step growth process of functional monomers. When preparing vitrimers from olefin or vinyl monomers, three main synthetic approaches can be followed: (a) the direct copolymerization of such monomers with a multifunctional cross-linker containing exchangeable group $(s) ;{ }^{11}$ (b) the crosslinking of thermoplastic copolymers carrying pendant exchangeable functions with (macro)molecules containing several complementary exchangeable groups; ${ }^{9,15}$ or (c) the direct transformation of non-functional "conventional" plastics into vitrimers by reactive processing. ${ }^{15}$ The latter approach is particularly appealing to design industrially relevant vitrimers, as it allows transforming commercial plastics into vitrimers with the need to change neither their already optimized syntheses nor their current processing. Such a synthetic approach requires to develop 
efficient grafting chemistries and to make use of dynamic bonds that are thermally and chemically compatible with reactive processing. Moreover, the exchangeable links should be sufficiently dynamic to yield vitrimers with viscosities compatible with extrusion for example. We recently showed that the metathesis of dioxaborolanes meets all these criteria and we were able to transform high-density polyethylene (HDPE) into a vitrimer via a two-step reactive extrusion process. Radical grafting of maleimides bearing dioxaborolane functions was achieved in a first step, which was followed by the crosslinking of the functional PE with a complementary bisdioxaborolane. ${ }^{15}$

In this study, we aimed at simplifying even further the transformation of HDPE into a vitrimer, by performing a singlestep reactive extrusion. Additionally, we chose to test nitroxides as grafting agents in order to expand the chemical toolbox available to prepare vitrimers by reactive processing. To this aim, we synthesized a monofunctional dioxaborolane nitroxide, which was used to study the grafting process, as well as a bisnitroxide dioxaborolane, which was used to transform HDPE into a vitrimer by reactive extrusion. Vitrimers with different cross-linking densities were successfully prepared and fully characterized, paying special attention to their flow properties as a function of temperature, thermal stability, mechanical properties and recyclability.

\section{Experimental}

\section{Materials}

All chemical products and solvents were used as received and without further purification. Epichlorohydrin, tetrabutylammonium hydrogensulfate, oxalyl chloride, high density polyethylene (HDPE) $\left(M_{\mathrm{n}}=11 \mathrm{~kg} / \mathrm{mol}, \emptyset=7.5\right.$, melt index 2.2 $\mathrm{g} / 10 \mathrm{~min}$ ) and dicumyl peroxide (DCP) were purchased from Sigma-Aldrich. 4-Hydroxy-2,2,6,6-tetramethylpiperidine 1-oxyl (HO-TEMPO) and phenylboronic acid were purchased from $\mathrm{TCl}$. 1,4-phenylenebisboronic acid was purchased from Activate Scientific.

\section{Methods}

Differential scanning calorimetry (DSC). DSC analysis were performed on a TA DSC 250 apparatus. Two heating cycles, from -100 to $200{ }^{\circ} \mathrm{C}$, were recorded at $10^{\circ} \mathrm{C} / \mathrm{min}$. The values of enthalpy and melting temperature were measured on the second cycle.

Thermogravimetric Analyses (TGA). TGA analyses were conducted on a Netzsch TG 209 F1 Libra. Samples were submitted to a heating ramp from $25^{\circ} \mathrm{C}$ to $600^{\circ} \mathrm{C}$ at a speed of $10^{\circ} \mathrm{C} / \mathrm{min}$ under a nitrogen flow.

Dynamic mechanical analysis (DMA). DMA analyses were conducted on a TA Q800 apparatus in the film tension geometry with rectangular samples of $5 \mathrm{~mm} \times 1.5 \mathrm{~mm}$ cross section. Heating ramps were applied from $25^{\circ} \mathrm{C}$ to $220^{\circ} \mathrm{C}$ at a speed of $3{ }^{\circ} \mathrm{C} / \mathrm{min}$ with a maximum strain amplitude of $1 \%$ at fixed frequency of $1 \mathrm{~Hz}$.
Tensile tests. Uniaxial tensile tests were performed on an Instron 5564 apparatus with dumbbell-shaped geometries (gauge length $10 \mathrm{~mm}$ ) at a fixed crosshead speed of $10 \mathrm{~mm} / \mathrm{min}$. Tensile creep tests were performed on the same machine at $80^{\circ} \mathrm{C}$ and with same sample geometries. After $5 \mathrm{~min}$ of equilibration at $80^{\circ} \mathrm{C}$ a constant crosshead speed of $10 \mathrm{~mm} / \mathrm{min}$ was applied until the engineering stress reached $5 \mathrm{MPa}$. Then, this stress was maintained for $60000 \mathrm{~s}$ (Fig S.20). The creep values reported in Fig. 7 correspond to the increase in creep deformation between $10000 \mathrm{~s}$ and $60000 \mathrm{~s}$. These values were averaged over 4 tests on different specimens for reproducibility.

Rheological characterizations. Rheological measurements were performed on an Anton Paar MCR501 rheometer equipped with a convection oven under nitrogen flow using a parallel plate geometry (diameter $25 \mathrm{~mm}$ ). Stress relaxation measurements were carried out from $150^{\circ} \mathrm{C}$ to $250^{\circ} \mathrm{C}$ by applying a constant shear strain of $1 \%$. Zero shear viscosities were calculated from stress relaxation experiments according to the formula $\eta=\int_{0}^{\infty} G(t) d t$. Strain sweep measurements were performed with a strain amplitude ranging from 0.1 to 2 $\%$, with a constant angular frequency of $1 \mathrm{rad} / \mathrm{s}$.

Fourier-transform infrared (FTIR) spectroscopy. FTIR spectroscopy was conducted on a Tensor 37 spectrometer from Bruker in solid state and recorded in attenuated total reflectance (ATR) mode.

Nuclear magnetic resonance (NMR) spectroscopy. ${ }^{1} \mathrm{H}$ and ${ }^{13} \mathrm{C}$ NMR spectra were recorded at $297 \mathrm{~K}$ on a Bruker AVANCE 400 spectrometer at $400 \mathrm{MHz}$ and $100 \mathrm{MHz}$, respectively, and referenced to the residual solvent peaks $\left({ }^{1} \mathrm{H}, \delta 7.26\right.$ for $\mathrm{CDCl}_{3}$; ${ }^{13} \mathrm{C}, \delta 77.16$ for $\mathrm{CDCl}_{3}$ ). In the case of TEMPO derivatives, NMR analysis was run in the presence of phenylhydrazine, which was used to reduce the nitroxide function.

\section{Synthesis}

The synthesis of glycidyl-TEMPO (G-TEMPO), 2, 4-(2,3dihydroxypropoxy)-TEMPO (DHP-TEMPO), 3, and $\operatorname{Bis}(2,2,6,6-$ tetramethyl-4-piperidyl)-N,N'-dioxyl oxalate, Bis-TEMPO, 6, were carried out according to literature procedures. ${ }^{21-23}$

2,2,6,6-tetramethyl-4-((2-phenyl-1,3,2-dioxaborolan-4-yl)methoxy)piperidin-1-oxyl (TEMPO-BE), 4. 3-((1-oxyl-2,2,6,6tetramethylpiperidin-4-yl)oxy)propane-1,2-diol (0.34 g, 1.38 mmol, 1.0 eq.) was dissolved in THF $(5 \mathrm{~mL})$, and then phenylboronic acid (177 mg, $1.45 \mathrm{mmol}, 1.05$ eq.) was added followed by water $(0.5 \mathrm{~mL})$. The mixture was stirred 20 minutes before $\mathrm{MgSO}_{4}$ was added (498 $\mathrm{mg}$, 3eq.). The mixture was stirred overnight at room temperature. Then, the mixture was filtrated and the solvent was removed. TEMPO-BE, 4, was obtained as a red oil $(0.39 \mathrm{~g}, 85 \%)$. ${ }^{1} \mathrm{H} \mathrm{NMR}\left(\mathrm{CDCl}_{3}\right.$ with phenylhydrazine, $400 \mathrm{MHz}): \delta(\mathrm{ppm}): 7.80-7.70(\mathrm{~m}, 2 \mathrm{H}), 7.41-$ $7.35(\mathrm{~m}, 1 \mathrm{H}), 7.31-7.25(\mathrm{~m}, 2 \mathrm{H}), 4.64-4.55(\mathrm{~m}, 1 \mathrm{H}), 4.30(\mathrm{dd}, 1 \mathrm{H}$, $\mathrm{J}=9.1 \mathrm{~Hz}, \mathrm{~J}=8.2 \mathrm{~Hz}$ ), $4.07(\mathrm{dd}, 1 \mathrm{H}, \mathrm{J}=9.1 \mathrm{~Hz}, \mathrm{~J}=6.4 \mathrm{~Hz}), 3.62-$ $3.52(\mathrm{~m}, 2 \mathrm{H}), 3.47(\mathrm{dd}, 1 \mathrm{H}, \mathrm{J}=10.2 \mathrm{~Hz}, \mathrm{~J}=5.1 \mathrm{~Hz}), 1.95-1.75(\mathrm{~m}$, $2 \mathrm{H}), 1.50-1.35(\mathrm{~m}, 2 \mathrm{H}), 1.20-1.00(\mathrm{~m}, 12 \mathrm{H})$ (Fig. $\mathrm{S} 1) .{ }^{13} \mathrm{C} \mathrm{NMR}$ $\left(\mathrm{CDCl}_{3}\right.$ with phenylhydrazine, $\left.100 \mathrm{MHz}\right): \delta$ (ppm): 156.03, $134.79,131.50,129.50,128.28,127.78,120.20,115.40,76.23$, 71.19, 70.17, 68.30, 62.64, 43.01, 21.70 (Fig. S2). 
4,4'-(((1,4-phenylenebis(1,3,2-dioxaborolane-2,4-diyl))bis(methylene))bis(oxy))bis(2,2,6,6-tetramethyl-piperidin-1-

oxyl) (Bis-TEMPO-BE), 5. 3-((1-oxyl-2,2,6,6-tetramethylpiperidin-4-yl)oxy)propane-1,2-diol (2.00 g, $8.12 \mathrm{mmol}, 2.0 \mathrm{eq}$.) was dissolved in THF (40 mL), and then 1,4-phenylenebisboronic acid $(673 \mathrm{mg}, 4.06 \mathrm{mmol}, 1.0$ eq.) was added followed by water $(1.5 \mathrm{~mL})$. The mixture was stirred 20 minutes before $\mathrm{MgSO}_{4}$ was added ( $1.47 \mathrm{~g}, 3$ eq.). The mixture was stirred overnight at room temperature. Then, the mixture was filtrated and the solvent was removed. Bis-TEMPO-BE, 5, was obtained as a red oil (1.05 g, 44\%). ${ }^{1} \mathrm{H} N M R\left(\mathrm{CDCl}_{3}\right.$ with phenylhydrazine, $\left.400 \mathrm{MHz}\right): \delta$ (ppm): 7.75-7.70 (m, 4H), 4.65-4.55 (m, 2H), $4.30(\mathrm{dd}, 2 \mathrm{H}, \mathrm{J}=9.0$ $\mathrm{Hz}, \mathrm{J}=8.2 \mathrm{~Hz}), 4.08(\mathrm{dd}, 2 \mathrm{H}, \mathrm{J}=9.0 \mathrm{~Hz}, \mathrm{~J}=6.4 \mathrm{~Hz}), 3.62-3.45(\mathrm{~m}$,

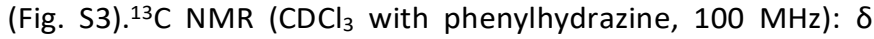
(ppm): 136.78, 134.02, 128.27, 115.07, 76.31, 71.36, 70.06, 68.34, 68.20, 43.54, 21.56 (Fig. S4).

Functionalization of the polyethylene. The functionalization and the cross-linking of polyethylene were carried out by reactive extrusion using a DSM Explore batch twin-screw extruder (5 $\mathrm{cm}^{3}$ capacity) equipped with co-rotating conical screw profile and recirculation channel to control the residence

A
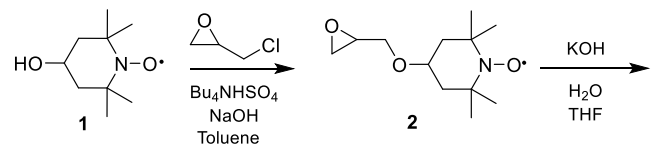<smiles>CC(O)CO</smiles>
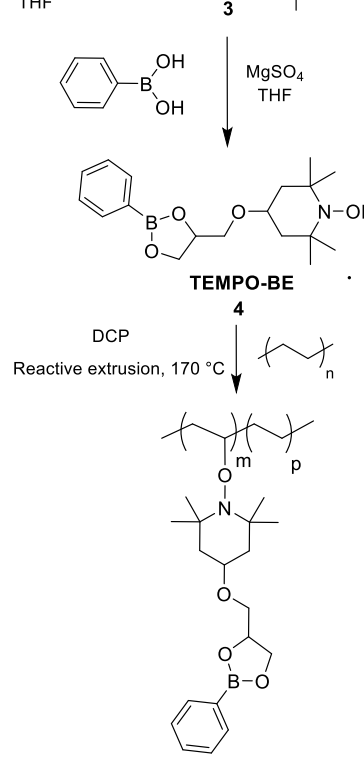

Grafted PE

PE-G $6 \mathrm{H}), 1.93-1.80(\mathrm{~m}, 4 \mathrm{H}), 1.55-1.40(\mathrm{~m}, 4 \mathrm{H}), 1.20-1.00(\mathrm{~m}, 24 \mathrm{H})$

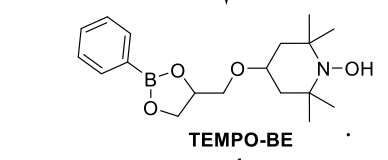

time. First polyethylene, TEMPO-BE or Bis-TEMPO-BE compounds and dicumyl peroxide ( $50 \% \mathrm{~mol}$ vs NO group) were mixed together prior to introduction in the extruder. The grafting was performed under nitrogen with a barrel temperature of $170{ }^{\circ} \mathrm{C}$ and a screw speed of $100 \mathrm{rpm}$. After 12 minutes, the polymer was driven out the extruder. The grafted polyethylene was used without purification for all characterizations, except to calculate the grafting efficiency, for which the grafted polymers were characterized by FTIR without purification as well as after washing.

Synthesis of model cross-linked polyethylene network, PE-X. Polyethylene, $0.6 \mathrm{~mol} \%$ of Bis-TEMPO, 6 , and $0.6 \mathrm{~mol} \%$ of dicumyl peroxide were mixed together in the extruder for $1 \mathrm{~min}$ with a barrel temperature of $170^{\circ} \mathrm{C}$ and a screw speed of 100 rpm. The mixture was then compression molded at $170{ }^{\circ} \mathrm{C}$ for $12 \mathrm{~min}$.

Samples preparation. All specimens were made by compression molding at $150^{\circ} \mathrm{C}$ for 7 minutes. Various geometries were used: rectangular shape for DMA, disc shape for rheology and dumbbell-shape for tensile tests.
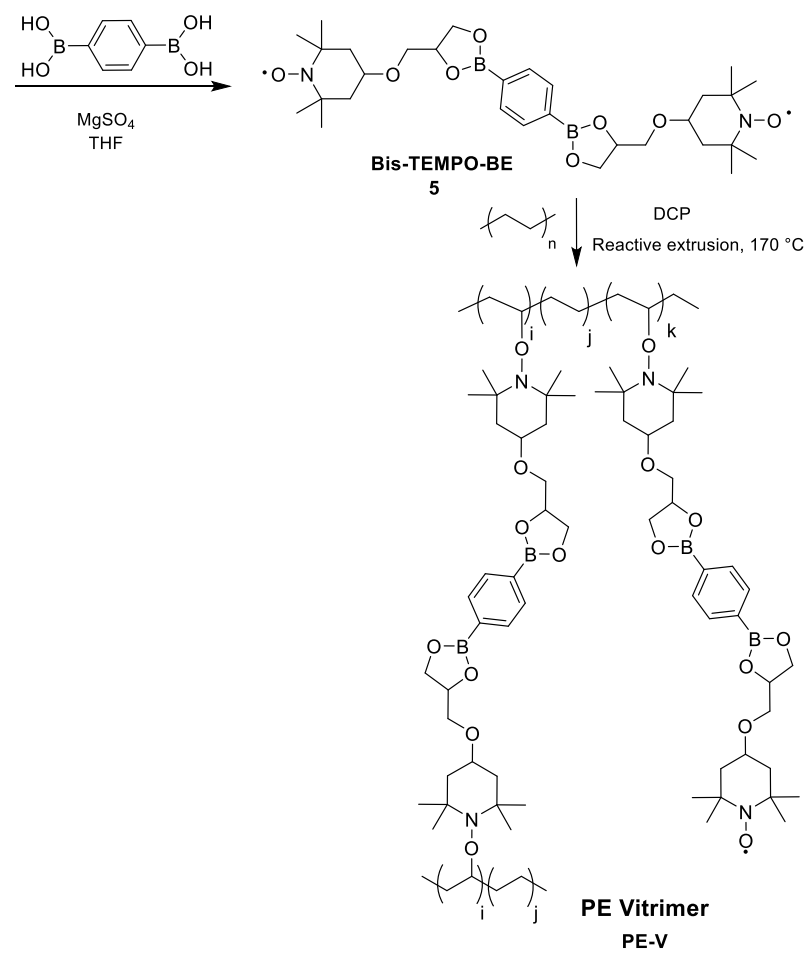

B

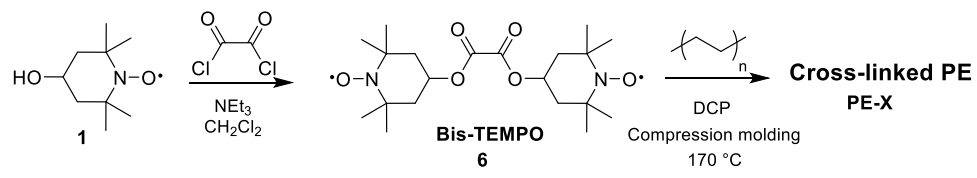

Scheme 1 (A) Synthesis of the grafting agents TEMPO-BE, 4, and Bis-TEMPO-BE, 5, and preparation of grafted-PE (PE-G) and PE vitrimer (PEV) by reactive extrusion of HDPE with $\mathbf{4}$ and $\mathbf{5}$, respectively. (B) Synthesis of the grafting agent Bis-TEMPO, 6, and preparation of cross-linked $\mathrm{PE}(\mathrm{PE}-\mathrm{X})$ by reactive compression molding of $\mathrm{PE}$ with $\mathbf{6}$. 


\section{Results and discussion}

\section{PE grafting}

Preparing polyolefins incorporating comonomers with polar functions is challenging, ${ }^{24}$ yet such polar monomers can bring improved properties to polyolefin plastics, such as adhesion, dyeability, paintability, wettability, compatibility with other polymers, ${ }^{25,26}$ or chemical modifiability to access vitrimers. ${ }^{15}$ As a result, alternative approaches, such as radical grafting by reactive extrusion, have been developed over the years. ${ }^{26,27}$ One of the main advantages of polyolefin functionalization by reactive processing is that it is performed without solvents, thereby potentially suppressing the need for precipitation and drying steps after chemical modification. This characteristic is extremely appealing from both an environmental and economical perspective. One of the main drawback of radical grafting of PE is radical termination through recombination, which yields permanent non-dynamic cross-links that significantly impact the viscosity of the materials and consequently their processability. ${ }^{26,27}$ Taking into account that turning thermoplastics into vitrimers is impacting the viscosity of the melt by transforming finite polymer chains into a chemical network, developing grafting approaches that give access to boronic ester-based vitrimers with minimal nondynamic cross-links is essential. Nitroxide radical coupling is therefore an attractive route to prepare HDPE vitrimers by reactive processing. Indeed, nitroxides are extremely reactive towards carbon-centered radicals, with which they react to generate thermoreversible alkoxyamines. As a consequence, they are commonly used as radical inhibitors, antioxidants, polymerization mediators, ${ }^{28,29}$ and more recently grafting agents. ${ }^{30-33}$

A boronic ester bearing a single nitroxide function, TEMPO$\mathrm{BE}$, and a bis-boronic ester carrying two nitroxide functions, BisTEMPO-BE, were synthesized to be used as a model molecule to study PE grafting and to prepare HDPE vitrimer by reactive extrusion, respectively (Scheme 1). These two TEMPO derivatives were obtained in only three steps, starting from commercially available HO-TEMPO, 1. First, HO-TEMPO was subjected to a nucleophilic substitution with epichlorohydrin to obtain G-TEMPO, 2 . Then, the epoxide ring was hydrolyzed in basic media to get DHP-TEMPO, 3. Finally, TEMPO-BE, 4, was obtained by condensation of DHP-TEMPO on phenylboronic acid, while the Bis-TEMPO-BE cross-linker, 5, was obtained by condensation of DHP-TEMPO on 1,4-phenylenebisboronic acid.

The functionalization of HDPE was first studied with TEMPO$\mathrm{BE}$ in order to precisely quantify the grafting efficiency as a function of the mol\% of boronic ester reactive additive $[(\mathrm{mol}$ of $\mathrm{BE}$ additive per mol of ethylene repeating unit) $\times 100]$, which ranged from $0.45 \%$ to $1.84 \%$ (Table 1 ). Grafting was carried out at $170{ }^{\circ} \mathrm{C}$ for $12 \mathrm{~min}$ by reactive extrusion of commercial polyethylene with dicumyl peroxide (DCP) as the radical initiator. For the entire study, a constant molar ratio of 0.5 was kept between DCP and the NO functions of TEMPO-BE and BisTEMPO-BE in order to limit the formation of non-dynamic crosslinks caused by the recombination of macroradicals. The radical grafting of TEMPO-BE onto PE was quantified by FTIR spectroscopy using calibration curves based on physical mixtures obtained by melt extrusion of TEMPO-BE and PE without DCP (Figs. S5-S8). The grafting efficiency was determined by comparing the $\mathrm{B}-\mathrm{O}\left(1359 \mathrm{~cm}^{-1}\right)$ and $\mathrm{B}-\mathrm{C}(1100$ $\mathrm{cm}^{-1}$ ) vibration bands of TEMPO-BE to the $\mathrm{C}-\mathrm{H}$ bending vibration $\left(1470 \mathrm{~cm}^{-1}\right)$ of PE in the as-extruded blends and the washed polymers. For lower molar ratios of TEMPO-BE, e.g. 0.45 and 0.7 mol\%, a grafting efficiency of ca. $75 \%$ was obtained, which decreased to ca. $50 \%$ when the mol\% of TEMPO-BE was comprised between $1 \%$ and $2 \%$ (Table 1 ). Interestingly, all PE samples grafted with TEMPO-BE were completely soluble in xylene at $110{ }^{\circ} \mathrm{C}$, indicating that coupling reactions between $\mathrm{PE}$ macroradicals were sufficiently low under these conditions to prevent gelation (Table 1).

Table 1 Grafting yields and gel content of grafted PE (PE-G) and PE vitrimers $(\mathrm{PE}-\mathrm{V})$ prepared by reactive extrusion of $\mathrm{PE}$ at $170^{\circ} \mathrm{C}$ in the presence of TEMPO-BE and Bis-TEMPO-BE grafting agents, respectively.

\begin{tabular}{|c|c|c|c|c|}
\hline Sample & $\begin{array}{l}\text { NO group in } \\
\text { as extruded } \\
\text { blend } \\
(\% \mathrm{~mol})\end{array}$ & $\begin{array}{l}\text { NO-grafted } \\
\text { in washed } \\
\text { polymer } \\
\text { (\%mol) }\end{array}$ & $\begin{array}{l}\text { Grafting } \\
\text { yield (\%) }\end{array}$ & $\begin{array}{c}\text { Gel } \\
\text { content } \\
(\%)\end{array}$ \\
\hline PE-G1 ${ }^{a}$ & 0.45 & 0.34 & 76 & 0 \\
\hline $\mathrm{PE}-\mathrm{G} 2^{a}$ & 0.69 & 0.53 & 77 & 0 \\
\hline PE-G3 ${ }^{a}$ & 1.02 & 0.51 & 50 & 0 \\
\hline PE-G4 ${ }^{a}$ & 1.84 & 0.95 & 52 & 0 \\
\hline PE-V1 $b$ & 0.86 & 0.45 & 52 & 20 \\
\hline PE-V2 ${ }^{b}$ & 1.44 & 0.77 & 53 & 32 \\
\hline
\end{tabular}

\section{Vitrimer synthesis}

Based on these promising results, two routes can be followed to prepare PE vitrimers by reactive processing. The first one is the addition of a bis-dioxaborolane cross-linker once radical grafting of TEMPO-BE is complete. In that case, cross-linking takes place through dioxaborolane metathesis between the pendant BE moieties grafted on PE and the dioxaborolane functions of the cross-linker. ${ }^{15}$ This route is thus a two-step process, i.e., grafting and subsequent cross-linking, during which two small boronic ester molecules are generated within 
the vitrimer network for every cross-linking event. The second route relies on the reactive grafting of Bis-TEMPO-BE, which could yield vitrimers in a single step without releasing small molecules into the network. Although appealing, such a direct procedure has yet to be exemplified. Consequently, we opted for the second route and attempted to prepare vitrimers by reactive processing of HDPE in the presence of 0.43 and 0.72 mol\% of Bis-TEMPO-BE (Table 1, PE-V1 and PE-V2, respectively). While extrudable, both materials could not be dissolved in xylene at $110^{\circ} \mathrm{C}$, indicating that PE was successfully crosslinked. Gel contents of 20 and $32 \%$ were obtained when PE was extruded in the presence of 0.4 and 0.7 mol\% of Bis-TEMPO-BE, respectively. Although relatively low, these gel contents are perfectly comparable to those obtained on the same PE with a two-step procedure consisting of radical grafting of maleimides bearing dioxaborolane functionalities followed by cross-linking with a bis-dioxaborolane. ${ }^{15,34}$ Such gel contents reflect the relatively low molar mass of the HDPE precursor $\left(M_{n}=11\right.$ $\left.\mathrm{kg} / \mathrm{mol}, \mathrm{DP}_{\mathrm{n}}=390\right)$, its broad dispersity $(\theta=7.5)$ and the low ratio of Bis-TEMPO-BE used to cross-link. To confirm the absence of permanent non-dynamic cross-links in the vitrimers, the insoluble parts were swollen in xylene at $110^{\circ} \mathrm{C}$ and $1,2-$ butane-diol was added to selectively diolyze the boronic ester cross-links. After 15 minutes, all samples became entirely soluble, confirming that the materials were cross-linked with dynamic dioxaborolanes.

The grafting efficiency was quantified by FTIR spectroscopy on the insoluble part, which was further washed with hot xylene and dried under vacuum before measurement. Grafting yields of ca. $50 \%$ were found for both PE-V1 and PE-V2 samples (Table 1 , Fig. S9), indicating that the grafting efficiency of Bis-TEMPO$B E$ was slightly lower than that of TEMPO-BE, as is expected when comparing difunctional and monofunctional grafting agents. The influence of the degree of grafting on HDPE crystallinity was studied by differential scanning calorimetry (Fig. S11). Both vitrimers exhibit similar melting points as the polyethylene precursor, going from $128^{\circ} \mathrm{C}$ for the starting HDPE to 127 and $124^{\circ} \mathrm{C}$ for PE-V1 and PE-V2, respectively (Table S1). The degree of crystallinity of the materials was more significantly impacted by the chemical grafting, with a degree of crystallinity decreasing from $64 \%$ for the starting HDPE to 52 and $44 \%$ for PE-V1 and PE-V2, prepared with 0.43 and 0.72 mol\% of Bis-TEMPO-BE, respectively.

\section{Vitrimer flow properties}

The cross-linked nature of the $\mathrm{PE}$ vitrimers prepared by reactive extrusion of HDPE with Bis-TEMPO-BE was further confirmed by dynamic mechanical analyses. Figure 1 shows that the starting HDPE behaves like a typical semi-crystalline thermoplastic, with an elastic modulus ( $E^{\prime}$ ) decreasing abruptly above its melting temperature. Above the melting temperature, the virgin HDPE flows under its own weight, leading to the rupture of the sample. While the elastic modulus of the vitrimers and the starting HDPE follow a very similar trend between room and melting temperatures, being predominantly governed by the crystalline phase of the materials, the E' of vitrimers displays a very different behavior above $140{ }^{\circ} \mathrm{C}$. Thanks to their cross- linked nature, vitrimers exhibit an improved melt strength with the appearance of an elastomeric plateau, whose value is dependent on the cross-linking density, ca. 0.40 and $0.94 \mathrm{MPa}$ at $160^{\circ} \mathrm{C}$ for PE-V1 and PE-V2, respectively.

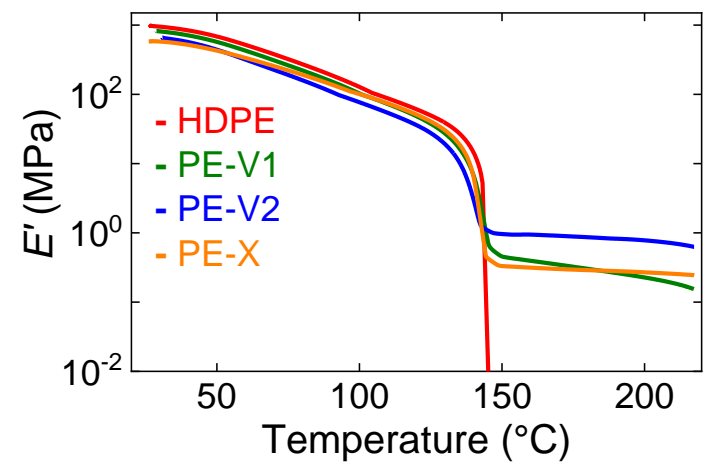

Fig.1 Dynamic mechanical analysis, in tension film geometry $(1 \mathrm{~Hz})$, of the HDPE precursor (red), PE vitrimers PE-V1(blue) and PE-V2 (green), and cross-linked PE, PE-X (orange).

Although permanently cross-linked, HDPE vitrimers flow at high temperature because of the reshuffling of boronic ester crosslinks via dioxaborolane metathesis. This characteristic is an essential feature of vitrimers, as it is responsible for their processability and recyclability.

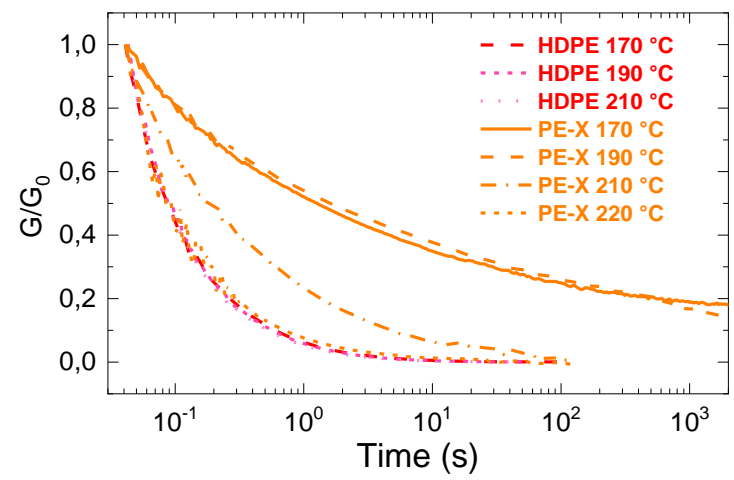

Fig. 2 Normalized stress relaxation profiles (1\% strain) of the HDPE precursor (red) and cross-linked model network, PE-X (orange).

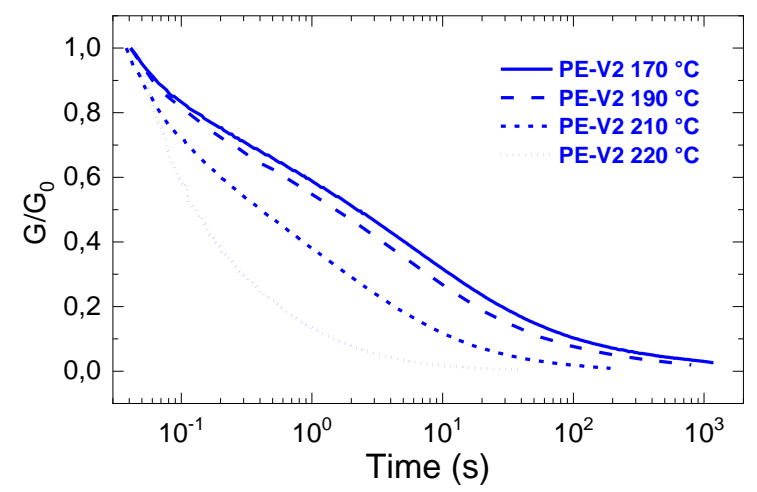

Fig. 3 Normalized stress relaxation profiles ( $1 \%$ strain) of the vitrimer $\mathrm{PE}-\mathrm{V} 2$ at different temperatures.

To further probe this behavior, the viscosity of the vitrimers was determined at different temperatures (between $170^{\circ} \mathrm{C}$ and $220^{\circ} \mathrm{C}$ ) through stress relaxation experiments using a rheometer 
equipped with a parallel plate geometry and a constant shear strain of $1 \%$. The polyethylene precursor, as well as the model network, PE-X, were also characterized for comparison (Figs. 2, 3 and S12). The model network was prepared by grafting onto the HDPE precursor, 0.6 mol\% of Bis-TEMPO, 6, a bis-nitroxide cross-linker containing no exchangeable boronic ester moiety (Scheme 1). DMA analysis of the model network PE-X confirmed that this material has a cross-linking density comparable to that of PE-V1 (Fig. 1). Stress relaxation experiments on HDPE at $170^{\circ} \mathrm{C}, 190^{\circ} \mathrm{C}$ and $210^{\circ} \mathrm{C}$, yielded almost identical results. The semi-crystalline thermoplastic completely relaxed stress within ca. $10 \mathrm{~s}$ (Fig. 2), with a zero shear viscosity only slightly decreasing from $1.2 \times 10^{4}$ Pa.s to $6.14 \times 10^{4} \mathrm{~Pa}$.s going from $170^{\circ} \mathrm{C}$ to $210^{\circ} \mathrm{C}$ (Table S2).

In contrast, the evolution of the relaxation modulus as a function of time was significantly impacted by the temperature for both vitrimers (Figs. 3 and S12, for PE-V2 and PE-V1, respectively). Up to $210{ }^{\circ} \mathrm{C}, \mathrm{PE}-\mathrm{V} 2$ vitrimer relaxed stress much slower than polyethylene, with relaxation times progressively decreasing with temperatures. However, both vitrimers relaxed stress much more rapidly above $210^{\circ} \mathrm{C}$, with complete relaxation occurring in less than $30 \mathrm{~s}$, very similarly to the HDPE precursor. This translates in a singular evolution of the viscosity as a function of temperature (Fig. 4, Table S2). The viscosity of the PE-V2 vitrimer decreases progressively from $1.66 \times 10^{7} \mathrm{~Pa} . \mathrm{S}$ to $1.01 \times 10^{6} \mathrm{~Pa}$.s between $170^{\circ} \mathrm{C}$ and $210^{\circ} \mathrm{C}$, before dropping abruptly to $2.54 \times 10^{4} \mathrm{~Pa}$.s at $220^{\circ} \mathrm{C}$, a value comparable to that of HDPE. A very similar trend was observed with PE-V1, the drop of viscosity taking place $10^{\circ} \mathrm{C}$ lower (Fig. 4, Table S2). This abrupt decrease of the viscosity above $200-210^{\circ} \mathrm{C}$ is likely a consequence of the dissociation of the thermolabile alkoxyamine bonds linking the dioxaborolane cross-links to the $P E$ backbone. Stress relaxation experiments performed at different temperatures on the non-dynamic PE network, $\mathrm{PE}-\mathrm{X}$, were consistent with this hypothesis (Fig. 2). Up to $190{ }^{\circ} \mathrm{C}$, the relaxation of $\mathrm{PE}-\mathrm{X}$ is not only slower than that of vitrimers $\mathrm{PE}-$ $\mathrm{V} 1$ and PE-V2, but is also incomplete, with a residual stress of $16 \%$ after $1000 \mathrm{~s}$. This indicates that the complete relaxation observed on vitrimers below $200^{\circ} \mathrm{C}$ is the result of boronic ester metathesis between cross-links. However, the stress relaxation behavior of $\mathrm{PE}-\mathrm{X}$ resembles that of the PE precursor at higher temperature, with a complete relaxation reached in approximately $10 \mathrm{~s}$ at $220^{\circ} \mathrm{C}$.

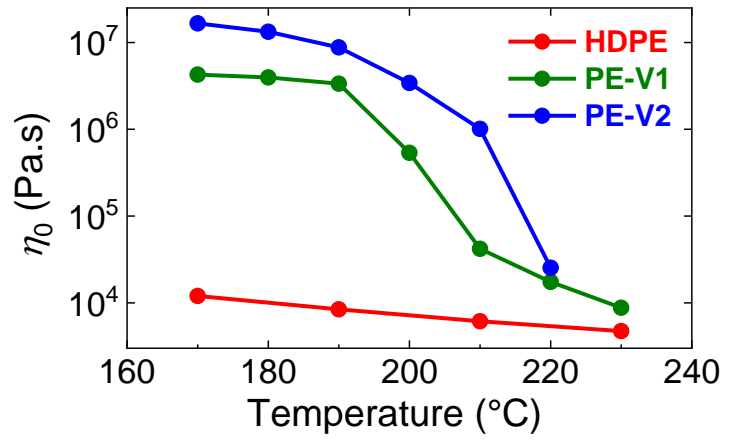

Fig. 4 Zero shear viscosities, $\eta_{0}$ (Pa.s), of HDPE, PE-V1 and PE-V2 vitrimers obtained from stress relaxation experiments.
To further confirm this hypothesis, solubility tests were performed on PE-V1 and PE-V2 annealed at $250^{\circ} \mathrm{C}$. Both materials were then entirely soluble in xylene after 15 minutes at $110^{\circ} \mathrm{C}$. The resulting soluble polymers were precipitated into acetone, dried and characterized by infrared spectroscopy. In both cases, the B-O $\left(1359 \mathrm{~cm}^{-1}\right)$ and $\mathrm{B}-\mathrm{C}\left(1100 \mathrm{~cm}^{-1}\right)$ vibration bands corresponding to the dioxaborolane functions of the BisTEMPO-BE cross-linker, 5, had entirely disappeared, confirming the complete and permanent detachment of the bis-TEMPO-BE cross-linker from the PE backbone after heating the material to $250{ }^{\circ} \mathrm{C}$ (Figs. S13 and S14).

Strain sweep experiments were also performed to better characterize the peculiar behavior of vitrimers as a function of temperature (Figs. 5 and S15-S17). These measurements were performed using a parallel plate geometry, applying a strain amplitude ranging from 0.1 to $2 \%$ and a constant angular frequency of $1 \mathrm{rad} / \mathrm{s}$. Between 150 and $190^{\circ} \mathrm{C}$, the storage $\left(\mathrm{G}^{\prime}\right)$ and loss ( $\left.G^{\prime \prime}\right)$ moduli of PE-V2 measured at a strain of $0.1 \%$ remained approximately constant around 140 and $40 \mathrm{kPa}$, respectively, confirming the network structure of the material. When PE-V2 was heated to $200^{\circ} \mathrm{C}$ and more, both moduli dropped, with G' still remaining greater than G" (Fig. 5, blue line and symbols). However, when PE-V2 was kept at $220^{\circ} \mathrm{C}$ for 40 min, both $G^{\prime}$ and $G^{\prime \prime}$ dropped significantly, with $G^{\prime \prime}$ now being superior to $G^{\prime}$, indicating that the material was below the gel point at this stage (Fig. 5, red symbols). A similar trend was observed for PE-V1 vitrimer, the gel-sol transition occurring at $210{ }^{\circ} \mathrm{C}$ (Figs. S16 and S17). This dissociation of the alkoxyamine link above $210^{\circ} \mathrm{C}$ proved to be irreversible. Indeed, when strain sweep experiments were performed at $170^{\circ} \mathrm{C}$ on vitrimer samples previously annealed at $220^{\circ} \mathrm{C}, \mathrm{G}^{\prime \prime}$ was superior to $\mathrm{G}^{\prime}$ over the entire strain amplitude, with values just slightly greater than those measured at $220^{\circ} \mathrm{C}$ for both moduli (Fig. 5, red symbols). These results further confirm the irreversible detachment of the bis-TEMPO-BE cross-linker when vitrimers are subjected to temperatures higher than $210-220^{\circ} \mathrm{C}$ for a long period of time.

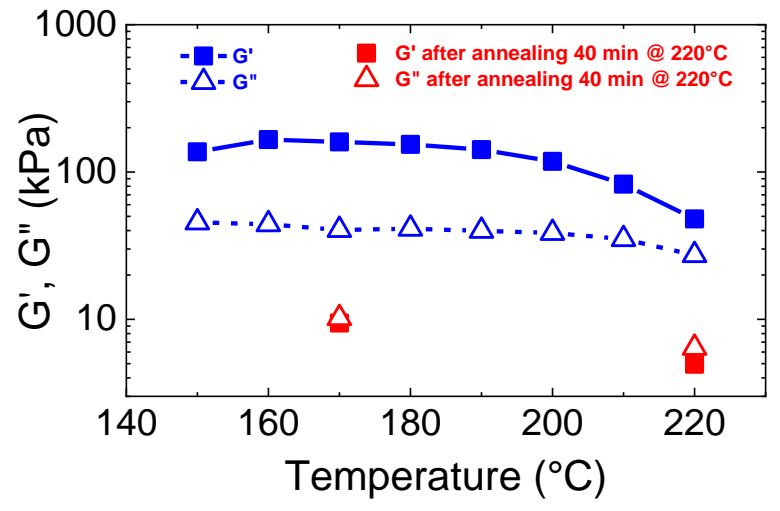

Fig. 5 Storage ( $G^{\prime}$, square) and loss ( $G^{\prime \prime}$, triangle) modulus of vitrimer $\mathrm{PE}-\mathrm{V} 2$ measured at an angular frequency of $1 \mathrm{rad}^{-\mathrm{s}^{-1}}$ and a strain of $0.1 \%$. Blue: at various temperatures. Red: at $220^{\circ} \mathrm{C}$ and $170^{\circ} \mathrm{C}$ after annealing $40 \mathrm{~min}$ at $220^{\circ} \mathrm{C}$. 
Vitrimer thermo-mechanical properties

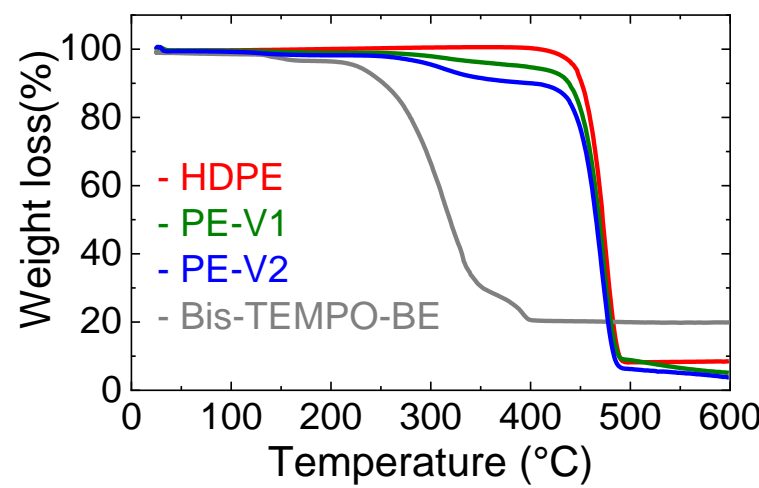

Fig. 6 Weight loss versus temperature of HDPE precursor, PE-V1 and $\mathrm{PE}-\mathrm{V} 2$ vitrimers, and Bis-TEMPO-BE cross-linker 5 measured by TGA.

The thermal stabilities of the PE-V1 and PE-V2 vitrimers and the HDPE precursor were assessed by thermogravimetric analysis (TGA), performing a heating ramp from $25^{\circ} \mathrm{C}$ to $600{ }^{\circ} \mathrm{C}$ (Fig. 6). The HDPE started to slowly lose weight above $400^{\circ} \mathrm{C}$, with an abrupt loss taking place as from $440^{\circ} \mathrm{C}$. PE-V1 and PEV2 vitrimers also showed an abrupt loss of weight as off $440{ }^{\circ} \mathrm{C}$. However, both materials lost some weight before this temperature, with a weight loss of 1.1 and $2.1 \mathrm{wt} \%$ at $250{ }^{\circ} \mathrm{C}$ and 5.3 and $10 \mathrm{wt} \%$ at $400{ }^{\circ} \mathrm{C}$ for PE-V1 and PE-V2 vitrimers, respectively. Interestingly, the weight loss at $400{ }^{\circ} \mathrm{C}$ observed for both materials matches well with the amount of ungrafted Bis-TEMPO-BE. These results indicate that despite the irreversible dissociation of the alkoxyamine bonds above $220^{\circ} \mathrm{C}$, vitrimers display very good thermal stability.

Table 2 Tensile properties at RT of HDPE and PE-V1 and PE-V2 vitrimers as processed and recycled (up to 3 times). Average on 3 samples

\begin{tabular}{cccc}
\hline & $\begin{array}{c}\text { Young's } \\
\text { modulus } \\
(\mathrm{MPa})\end{array}$ & $\begin{array}{c}\text { Tensile } \\
\text { strength (MPa) }\end{array}$ & $\begin{array}{c}\text { Elongation at } \\
\text { break (\%) }\end{array}$ \\
\hline HDPE & $495 \pm 22$ & $19.9 \pm 0.5$ & $1060 \pm 91$ \\
\hline PE-V1 as extruded & $444 \pm 20$ & $19.5 \pm 0.2$ & $675 \pm 42$ \\
PE-V1 recycled x1 & $403 \pm 29$ & $17.4 \pm 1.3$ & $583 \pm 99$ \\
PE-V1 recycled x2 & $418 \pm 30$ & $17.8 \pm 1.0$ & $492 \pm 53$ \\
PE-V1 recycled x3 & $408 \pm 24$ & $17.2 \pm 0.9$ & $621 \pm 27$ \\
\hline PE-V2 as extruded & $334 \pm 26$ & $15.3 \pm 1.1$ & $436 \pm 22$ \\
PE-V2 recycled x1 & $327 \pm 9$ & $14.9 \pm 0.3$ & $462 \pm 40$ \\
PE-V2 recycled x2 & $328 \pm 18$ & $16.1 \pm 1.0$ & $507 \pm 65$ \\
PE-V2 recycled x3 & $347 \pm 22$ & $16.6 \pm 0.5$ & $384 \pm 34$ \\
\hline
\end{tabular}

The mechanical properties of the vitrimers and the HDPE precursor were evaluated via tensile tests at $25^{\circ} \mathrm{C}$ (Table 2, Fig $\mathrm{S} 18)$. After extrusion, the Young's modulus and the tensile strength of PE-V1 vitrimer were comparable to that of HDPE, but were 33 and $23 \%$ lower, respectively, in the case of PE-V2 vitrimer, reflecting the lower degree of crystallinity of PE-V2
(Table S1). The elongation at break of the vitrimers decreased from $1060 \%$ for the HDPE precursor to 675 and $436 \%$ for PEV1 and PE-V2 vitrimers, respectively. This behavior is characteristic of cross-linked materials. However, a unique property of vitrimers is their ability to be recycled. Both PE-V1 and $\mathrm{PE}-\mathrm{V} 2$ could be processed, ground, and reprocessed several times by means of extrusion or compression molding. After three recycling cycles, the mechanical properties of vitrimers were unaltered, demonstrating the ability of these permanently cross-linked materials to be efficiently recycled (Table 2 ).

Tensile tests were also performed at $80{ }^{\circ} \mathrm{C}$ on the vitrimers and their HDPE precursor (Fig. S19). At this temperature, both vitrimers displayed a tensile strength comparable to HDPE, while their dimensional stability was further increased as compared to HDPE, whose elongation at break reached 1,960\%, compared to 785 and $530 \%$ for PE-V1 and PE-V2 vitrimers, respectively (Table 3 ). The creep resistance of these three polymers was evaluated at $80^{\circ} \mathrm{C}$ by placing these materials under a constant load of $5 \mathrm{MPa}$ for $60,000 \mathrm{~s}$. Vitrimers PE-V1 and PE-V2 both showed superior creep resistances, with an improvement of 20 and $33 \%$, respectively, as compared to HDPE (Figs. 7 and S20).

Table 3 Tensile properties at $80^{\circ} \mathrm{C}$ of HDPE and PE-V1 and PE-V2 vitrimers as processed and recycled (up to 3 times). Average on 3 samples

\begin{tabular}{cccc}
\hline & $\begin{array}{c}\text { Young's modulus } \\
(\mathrm{MPa})\end{array}$ & $\begin{array}{c}\text { Tensile strength } \\
(\mathrm{MPa})\end{array}$ & $\begin{array}{c}\text { Elongation at } \\
\text { break (\%) }\end{array}$ \\
\hline HDPE & $123 \pm 35$ & $6.07 \pm 0.54$ & $1960 \pm 197$ \\
PE-V1 & $94 \pm 8$ & $6.03 \pm 0.45$ & $784 \pm 65$ \\
PE-V2 & $59 \pm 8$ & $6.52 \pm 1.0$ & $530 \pm 41$ \\
\hline
\end{tabular}

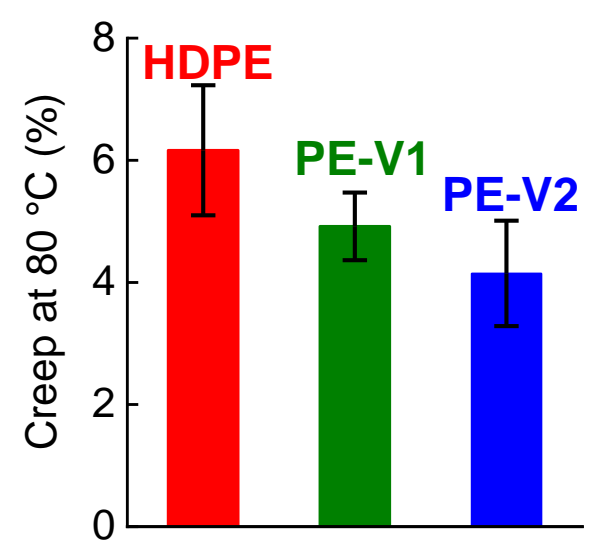

Fig. 7 Creep resistance at $80^{\circ} \mathrm{C}$ of PE vitrimers PE-V1 and PE-V2 as compared with the commercial HDPE precursor (4 specimens tested for each material).

\section{Conclusions}

The transformation of commercial HDPE into a vitrimer by reactive extrusion using a single-step procedure was investigated. To this aim, a bis-boronic ester carrying two 
nitroxide functions was prepared via a three-step synthetic route. Nitroxide chemistry was selected to graft the cross-linker in an attempt to limit as much as possible the formation of nondynamic cross-links caused by the recombination of macroradicals. Using less than one mol\% of bis-boronic ester cross-linker, HDPE vitrimers could be prepared by reactive extrusion. Vitrimers were able to completely relaxed stress at temperatures typically used to extrude HDPE, i.e., between $170^{\circ} \mathrm{C}$ and $200^{\circ} \mathrm{C}$. Using a model network prepared with a bisnitroxide that did not contain a dioxaborolane function, it was demonstrated that the complete stress relaxation observed below $200{ }^{\circ} \mathrm{C}$ for vitrimers was the result of dioxaborolane metathesis. It was also demonstrated that the alkoxyamine link formed as a result of the grafting of the bis-nitroxide crosslinker onto HDPE underwent irreversible detachment when vitrimers were exposed to temperatures superior to $210-220^{\circ} \mathrm{C}$ for prolonged periods of time. As a consequence, the zero-shear viscosity of these vitrimers follows a singular behavior as function of temperature. The viscosity decreases progressively with temperature between $160^{\circ} \mathrm{C}$ and approximately $200^{\circ} \mathrm{C}$, before dropping abruptly above $200-210^{\circ} \mathrm{C}$, to reach a viscosity comparable to that of the HDPE precursor. Therefore, below $200^{\circ} \mathrm{C}$, associative exchange reactions are mostly responsible for the flow properties of the vitrimers, while irreversible uncross-linking is predominant above $220^{\circ} \mathrm{C}$. This trend was confirmed and quantified via oscillatory rheology, performing strain sweep experiments. Thermogravimetric analyses of the vitrimers showed that these materials are thermally stable during heating ramps, with weight loss of 1 to $2 \%$ at $250{ }^{\circ} \mathrm{C}$. It was also demonstrated that the vitrimers can be re-processed multiple times without significant deterioration of their mechanical properties.

The approach developed allowed access to vitrimers from commercial HDPE via a single-step reactive extrusion. This method should be applicable to other polymers that can be modified by radical grafting, allowing to transform a significant range of thermoplastics into vitrimers without modifying their existing and optimized syntheses. In parallel, if vitrimers with a greater thermal stability of the crosslinks are aimed at, the TEMPO moiety could be advantageously replaced by $2,2,10,10$ tetramethylisoindolin- $\mathrm{N}$-oxyl (TMIO), which is thermally more stable than TEMPO and generates alkoxyamines with dissociation rate constants typically one order of magnitude lower than TEMPO based alkoxyamines. ${ }^{35}$

\section{Conflicts of interest}

There are no conflicts to declare.

\section{Acknowledgements}

We thank Antoine Breuillac for technical assistance with DMA, DSC, TGA and tensile test equipment. We thank Nathan Van Zee for his critical reading of the manuscript.

\section{References}

1. J. Hopewell, R. Dvorak and E. Kosior, Philos. Trans. R. Soc., $B, 2009,364,2115$.

2. L. Imbernon and S. Norvez, Eur. Polym. J., 2016, 82, 347.

3. W. Denissen, J. M. Winne and F. E. Du Prez, Chem. Sci., 2016, 7, 30 .

4. Z. P. Zhang, M. Z. Rong and M. Q. Zhang, Prog. Polym. Sci., 2018, 80, 39.

5. L. Zhang and S. J. Rowan, Macromolecules, 2017, 50, 5051.

6. M. M. Obadia, A. Jourdain, P. Cassagnau, D. Montarnal and E. Drockenmuller, Adv. Funct. Mater., 2017, 27, n/a.

7. D. Montarnal, M. Capelot, F. Tournilhac and L. Leibler, Science, 2011, 334, 965.

8. M. Capelot, M. M. Unterlass, F. Tournilhac and L. Leibler, ACS Macro Lett., 2012, 1, 789.

9. O. R. Cromwell, J. Chung and Z. Guan, J. Am. Chem. Soc., 2015, 137, 6492.

$10 . \quad$ T. F. Scott, A. D. Schneider, W. D. Cook and C. N. Bowman, Science, 2005, 308, 1615.

11. R. Nicolay, J. Kamada, A. Van Wassen and K. Matyjaszewski, Macromolecules, 2010, 43, 4355.

12. P. Taynton, K. Yu, R. K. Shoemaker, Y. Jin, H. J. Qi and W. Zhang, Adv. Mater. (Weinheim, Ger.), 2014, 26, 3938.

13. W. Denissen, G. Rivero, R. Nicolay, L. Leibler, J. M. Winne and F. E. Du Prez, Adv. Funct. Mater., 2015, 25, 2451.

14. Y.-X. Lu, F. Tournilhac, L. Leibler and Z. Guan, J. Am. Chem. Soc., 2012, 134, 8424.

15. M. Röttger, T. Domenech, R. van der Weegen, A. Breuillac, R. Nicolaÿ and L. Leibler, Science, 2017, 356, 62.

16. R. L. Snyder, D. J. Fortman, G. X. De Hoe, M. A. Hillmyer and W. R. Dichtel, Macromolecules (Washington, DC, U. S.), 2018, 51, 389.

17. M. M. Obadia, B. P. Mudraboyina, A. Serghei, D. Montarnal and E. Drockenmuller, J. Am. Chem. Soc., 2015, 137, 6078.

18. B. Hendriks, J. Waelkens, J. M. Winne and F. E. Du Prez, ACS Macro Lett., 2017, 6, 930.

19. Y. Nishimura, J. Chung, H. Muradyan and Z. Guan, J. Am. Chem. Soc., 2017, 139, 14881.

20. J. S. A. Ishibashi and J. A. Kalow, ACS Macro Lett., 2018, 7, 482.

21. Z. Jia, C. A. Bell and M. J. Monteiro, Macromolecules, 2011, 44, 1747.

22. K. Huang, J. Huang, M. Pan, G. Wang and J. Huang, J. Polym. Sci., Part A: Polym. Chem., 2012, 50, 2635.

23. R. Horng and S.-K. Lee, Appl. Mech. Mater., 2014, 440, 31.

24. A. Nakamura, S. Ito and K. Nozaki, Chem. Rev., 2009, 109, 5215.

25. T. C. Chung, Prog. Polym. Sci., 2002, 27, 39.

26. E. Passaglia, S. Coiai and S. Augier, Prog. Polym. Sci., 2009, 34, 911.

27. G. Moad, Prog. Polym. Sci., 1999, 24, 81.

28. L. Tebben and A. Studer, Angew. Chem., Int. Ed., 2011, 50, 5034.

29. J. Nicolas, Y. Guillaneuf, C. Lefay, D. Bertin, D. Gigmes and B. Charleux, Prog. Polym. Sci., 2013, 38, 63.

F. Cicogna, S. Coiai, E. Passaglia, I. Tucci, L. Ricci, F. Ciardelli and A. Batistini, J. Polym. Sci., Part A: Polym. Chem., 2011, 49, 781.

31. F. Cicogna, S. Coiai, P. Rizzarelli, S. Carroccio, C. Gambarotti, I. Domenichelli, C. Yang, N. T. Dintcheva, G. 
Journal Name

Filippone, C. Pinzino and E. Passaglia, Polym. Chem., 2014,

5, 5656.

32. S. Coiai, E. Passaglia and F. Cicogna, Polym. Int., 2019, 68, 27.

33. L. Lienafa, S. Monge, Y. Guillaneuf, B. Ameduri, D. Siri, D. Gigmes and J.-J. Robin, Eur. Polym. J., 2018, 109, 55.

34. R. G. Ricarte, F. Tournilhac and L. Leibler, Macromolecules, 2019, 52, 432.

35. S. Marque, C. Le Mercier, P. Tordo and H. Fischer, Macromolecules, 2000, 33, 4403. 